\title{
Existence of internal modes of sine-Gordon kinks
}

\author{
Niurka R. Quintero* and Angel Sánchez ${ }^{\dagger}$ \\ Grupo Interdisciplinar de Sistemas Complicados (GISC), Departamento de Matemáticas, Universidad Carlos III de Madrid, \\ Avenida de la Universidad 30, E-28911 Leganés, Madrid, Spain \\ Franz G. Mertens \\ Physikalisches Institut, Universität Bayreuth, D-95440 Bayreuth, Germany
}

(Received 24 March 2000)

\begin{abstract}
We study whether or not sine-Gordon kinks exhibit internal modes or "quasimodes." By considering the response of the kinks to ac forces and initial distortions, we show that neither intrinsic internal modes nor "quasimodes" exist in contrast to previous reports. However, we do identify a different kind of internal mode bifurcating from the bottom edge of the phonon band which arises from the discretization of the system in the numerical simulations, thus confirming recent predictions.
\end{abstract}

PACS number(s): 05.45.Yv, 02.30.Jr, 03.50.-z, 63.20.Pw

\section{INTRODUCTION}

Solitons, which were originally a concept arising in the study of integrable systems [1], have rapidly become a key paradigm in perturbed, nonintegrable systems describing many physical applications [2]. A very successful picture of soliton dynamics under different perturbations has resulted from the use of collective coordinate (CC) techniques [3]: In many instances, solitons behave basically like pointlike particles, and therefore their time evolution can be predicted by equations of motion which are ordinary differential equations. Nevertheless, along the last decade it has been progressively realized that internal degrees of freedom of the solitons play a crucial role in a number of problems: They govern resonant soliton (or solitary wave) collisions [4], give rise to nontrivial soliton-impurity interactions [5], and can be excited both by ac forces [6] and by thermal noise [7]. Therefore, it is very important to assess whether or not physically relevant solitons possess internal modes.

In this Rapid Communication we study solitons of the sine-Gordon (SG) equation, which arises in a diverse range of areas of physics, covering from crystal dislocation theory to Josephson junctions [1-3]. In spite of the fact that linear stability analysis leads to only one eigenvalue in the discrete spectrum, corresponding to a zero frequency Goldstone mode [2], Boesch and Willis [8] claimed that they found an internal "quasimode" above but close to the lower phonon band edge. They described this "quasimode" as a long-lived oscillation of the width of the SG kink. The possibility of such a mode had been suggested earlier by Rice [9], by means of a variational approach that reproduced with great accuracy the internal mode frequency of the $\phi^{4}$ model [1] and predicted the existence of a similar mode for the SG problem. To our knowledge, Ref. [8] is the only paper reporting the observation of this mode, although hints of its existence are scattered all over the soliton literature [10]. A few years later, it was theoretically found [11], within the

\footnotetext{
*Electronic address: kinter@math.uc3m.es

†Electronic address: anxo@math.uc3m.es

†Electronic address: franz mertens@theo.phy.uni-bayreuth.de
}

Rice approach, that the internal mode of the SG and the $\phi^{4}$ kinks could be excited by a constant external force or by an initial distortion; however, no numerical evidence was presented to confirm these results. Therefore, we decided to revisit the issue of the existence of the internal mode of the SG equation in order to give a definitive answer to it. Our approach, based in part on our earlier findings [6], overcomes the difficulty that the internal "quasimode" would lie within the phonon band by allowing us to look at driving frequencies well in the gap. As we will show below, this permits us to establish clearly that this putative internal mode does not exist. Furthermore, we do observe the excitation of a discreteness induced internal mode [12,13], whose frequency is in excellent agreement with recent predictions by Kevrekidis and Jones [14]. We substantiate these claims by briefly recalling our main analytical results in Sec. II, collecting our numerical results in Sec. III, and discussing our conclusions in Sec. IV.

\section{RESONANCES DUE TO ac FORCES}

We begin by considering the perturbed SG equation

$$
\phi_{t t}-\phi_{x x}=-\sin \phi+\epsilon \sin \left(\delta t+\delta_{0}\right),
$$

where $\epsilon, \delta$, and $\delta_{0}$ are the amplitude, frequency, and phase of the external periodic force. In [6] we analyzed analytically and numerically the above problem in the more general context of nonlinear (possibly damped) Klein-Gordon systems, which includes Eq. (1) as well as the $\phi^{4}$ model. Particularizing our results, obtained by means of the generalized traveling wave Ansatz [15] combined with the Rice Ansatz [9], we find that the momentum $P(t)$ and the width of the kink $l(t)$ (of a general nonlinear Klein-Gordon equation; specific constants for the SG case will be given below) obey the equations

$$
\frac{d P}{d t}=-q \epsilon \sin \left(\delta t+\delta_{0}\right), \quad P(t) \equiv \frac{M_{0} l_{0} \dot{X}}{l(t)},
$$




$$
\alpha\left(\dot{l}^{2}-2 l \ddot{l}\right)=\frac{l^{2}}{l_{0}^{2}}\left(1+\frac{P^{2}}{M_{0}^{2}}\right)-1,
$$

where $X(t)$ is the kink position. For SG, $q=2 \pi, M_{0}=8$, $l_{0}=1$ and $\alpha=\pi^{2} / 12$. The equation for the momentum is linear and can be solved exactly, yielding

$$
P(t)=\left[P(0)-\frac{q \epsilon \cos \left(\delta_{0}\right)}{\delta}\right]+\frac{q \epsilon}{\delta} \cos \left(\delta t+\delta_{0}\right) .
$$

We thus see that the term $P(t)^{2}$ in Eq. (3) involves two frequencies, $\delta$ and $2 \delta$. As shown in [6], for the $\phi^{4}$ kink this leads to dramatic resonance effects when the ac driving frequency $\delta$ is half the frequency $\Omega_{i}$ of the internal mode. Without resonant excitation of kink modes, the kink oscillates around its initial position as a pure pointlike particle, as shown analytically and numerically in [16]. However, as shown in [6] for the above parameters, in case an internal mode is excited as in the $\phi^{4}$ system, the system energy grows and eventually leads to chaotic motion of the kink. In addition, the resonance at $\Omega_{i} / 2$ turns out to be much stronger than that at $\Omega_{i}$, which makes it a convenient way to probe those modes: In case the SG kink would possess such a degree of freedom, we should observe similar phenomena. In [8], the frequency of the numerically observed "quasimode", was $\omega_{s}=1.004 \pm 0.001$ in our units, whereas Rice's theoretical analysis leads to [9] $\Omega_{R}=1.103$. In both cases, the corresponding half frequencies lie well within the gap; we can thus force the system at the predicted resonances without exciting much radiation which could mask any internal mode effects.

\section{NUMERICAL RESULTS}

In this section, we present the results of our numerical search for internal modes or "quasimodes" of the SG equation. We have computed the numerical solution of the perturbed SG equation (1) by using the Strauss-Vázquez scheme [17], the total length of the system being $L=100$, the steps being $\Delta x=0.05, \Delta t=0.005$ (or other values when indicated). Free boundary conditions were also used, and the final time in the simulations was 25000 , enough to detect any possible resonance [6].

\section{A. ac force}

First, we have perturbed our system with an ac force of amplitude $\epsilon=0.01$ and phase $\delta_{0}=\pi / 2$. We have used a SG kink at rest as initial condition. We have computed the energy (as defined in [17]) during the evolution, and then the mean value of the energy in the time interval $10000 \leqslant t$ $\leqslant 25000$, studying a range of values of $\delta$ around half of the possible internal mode frequencies. Figure 1 collects our results for the mean energy as a function of the frequency. We clearly see that the mean energy rapidly increases for some values of $\delta$ which do not coincide with any of the proposed internal modes or "quasimodes.' The first (leftmost) maximum lies below half the frequency of the lower phonon band edge; therefore, this one cannot be associated with a phonon mode and we will discuss its origin in detail below. As for the other peaks, Table I shows that we have been able to

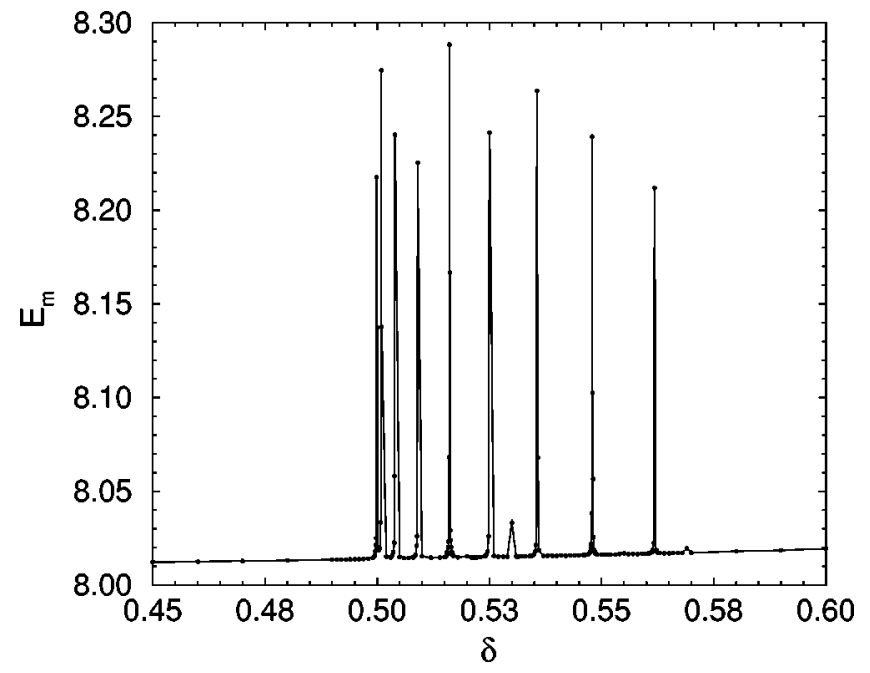

FIG. 1. Mean energy of the SG system, in dimensionless units, when $\delta$ is close to $\Omega_{R} / 2$. Points have been calculated from numerical simulations for $10000 \leqslant t \leqslant 25000$, with $\epsilon=0.01, \delta_{0}=\pi / 2$, $u(0)=0, X(0)=0$. The parameters of the discretization are $\Delta x$ $=0.05, \Delta t=0.005$, and $L=100$.

identify them with half of the values of the first frequencies of the phonon band, $\omega_{n}$, which are given by

$$
\omega_{n}=\sqrt{1+[(2 \pi n) / L]^{2}}, \quad n=1,2,3, \ldots, N=L / \Delta x .
$$

A comparison of the third and fourth columns of Table I, where we list the half values of the frequencies corresponding to the first eight radiational modes and the numerical peak frequencies $\widetilde{\omega}_{n}$ in Fig. 1, counting from the second maximum from the left, allows us to verify that these values are nearly identical in all cases (see also the relative differences $\left|\omega_{n} / 2-\tilde{\omega}_{n}\right|$ in Table I). We stress that identification of radiation modes as the origin of these resonances makes perfect physical sense: Indeed, an extended CC approach including radiation modes leads to the conclusion that the radiation modes should also be excited parametrically by ac drivings. In view of all this, the main conclusion we can draw from Fig. 1 is the absence of any resonance due to the internal quasimode in the phonon band reported by Boesch and Willis [8], which, in view of the frequency they reported, would lead to an extra peak located between the two right-

TABLE I. Comparison of the half of the first eight frequencies $\omega_{n} / 2$ of radiational modes with the computed frequencies $\tilde{\omega}_{n}$ from numerical simulations of Eq. (1) at which peaks in $E_{m}$ arise (see Fig. 1).

\begin{tabular}{ccccr}
\hline \hline $\operatorname{Mode}(n)$ & $\omega_{n}$ & $\omega_{n} / 2$ & $\tilde{\omega}_{n}$ & $\left|\omega_{n} / 2-\tilde{\omega}_{n}\right|$ \\
\hline 1 & 1.0019 & 0.5009 & 0.5009 & $8.6 \times 10^{-5}$ \\
2 & 1.0079 & 0.5039 & 0.5040 & $7 \times 10^{-5}$ \\
3 & 1.0176 & 0.5088 & 0.5090 & $1.9 \times 10^{-4}$ \\
4 & 1.0311 & 0.5155 & 0.5161 & $5.5 \times 10^{-4}$ \\
5 & 1.0482 & 0.5241 & 0.5250 & $9.1 \times 10^{-4}$ \\
6 & 1.0687 & 0.5343 & 0.5356 & $1.3 \times 10^{-3}$ \\
7 & 1.0924 & 0.5462 & 0.5479 & $1.7 \times 10^{-3}$ \\
8 & 1.1192 & 0.5596 & 0.5618 & $2.2 \times 10^{-3}$ \\
\hline \hline
\end{tabular}


most ones in Fig. 1. In this regard, it is important to note that the accuracy of our simulations in detecting every radiation mode rules out the possibility that we have missed this quasimode (at either $\omega_{s}$ or $\Omega_{R}$ ).

Interestingly, we do observe an internal mode: Let us recall that the first maximum of the energy occurs at $\delta=\omega_{d}$ $=0.4999$, i.e., $2 \omega_{d}=0.9998$ is below the lower phonon frequency $\omega_{p h}=1$ as we mentioned above. The reason for this peak is a different internal mode of the SG kink, that appears as a bifurcation from the lowest phonon of the continuum equation, of frequency $\omega_{p h}=1$, due to the effect of discreteness [12-14]. As has been recently shown [14], the frequency of this internal mode is $\omega_{i} \approx \sqrt{1-(4 / 2025)(\Delta x)^{4}}$ [the $(\Delta x)^{4}$ dependence was already observed in [12]]. In our simulations, $\Delta x=0.05$, hence $\omega_{i}=0.999998$, approximately equal to $2 \omega_{d}$. Therefore, the first peak in Fig. 1 corresponds to the parametric resonance at half the value of the discreteness induced internal mode, very much like the resonance with the $\phi^{4}$ kink internal mode reported earlier [6].

\section{B. Deformed initial kink}

In view of our negative result about the internal quasimode of the SG kink, we carried out more numerical simulations specifically designed to find it. Following [8], we chose initial conditions given by

$$
\begin{gathered}
\phi(x, 0)=4 \arctan \left(\exp \left[\frac{x-X(0)}{l(0)}\right]\right), \\
\phi_{t}(x, 0)=2 \frac{\left[-\frac{u(0)}{l(0)}-\frac{x-X(0)}{l^{2}(0)} l(0)\right]}{\cosh \left[\frac{x-X(0)}{l(0)}\right]},
\end{gathered}
$$

which correspond to a deformed kink if $l(0) \neq 1$ or $\dot{l}(0)$ $\neq 0$. By starting their simulations with such initial conditions, Boesch and Willis claimed that they were able to excite the internal quasimode, and hence we hope that, if it is indeed present, we should find it.

At this point, we have to recall the work of Majerníková, Gaididei, and Braun [11], who, as mentioned in the Introduction, theoretically considered the problem of a deformed kink given by the expressions above driven by a external constant force. When applied to this problem, our CC approach yields the same two ordinary differential equations as in [11] for $X(t)$ and $l(t)$ [Eqs. (2)-(3) without the sine term, i.e., with only a constant force $\epsilon]$. We have solved these equations exactly for arbitrary initial conditions [18] in terms of the Whittaker functions, concluding that the kink width oscillates only if $l(0) \neq l_{s}$. the natural width of the undistorted kink, $\left.1 / \sqrt{1-u(0)^{2}}\right]$ or $\dot{l}(0) \neq 0$ in Eq. (6), whereas external force and dissipation (if present) only damp out those oscillations. Hence, kink width oscillations should be easiest to detect without force and damping and, consequently, here we consider only free propagation of an initially distorted kink (a general analysis of the constant force problem will be presented elsewhere [18]). As a check of the validity of this procedure to probe internal modes, we have numerically verified that the $\phi^{4}$ kink indeed behaves in this fashion due

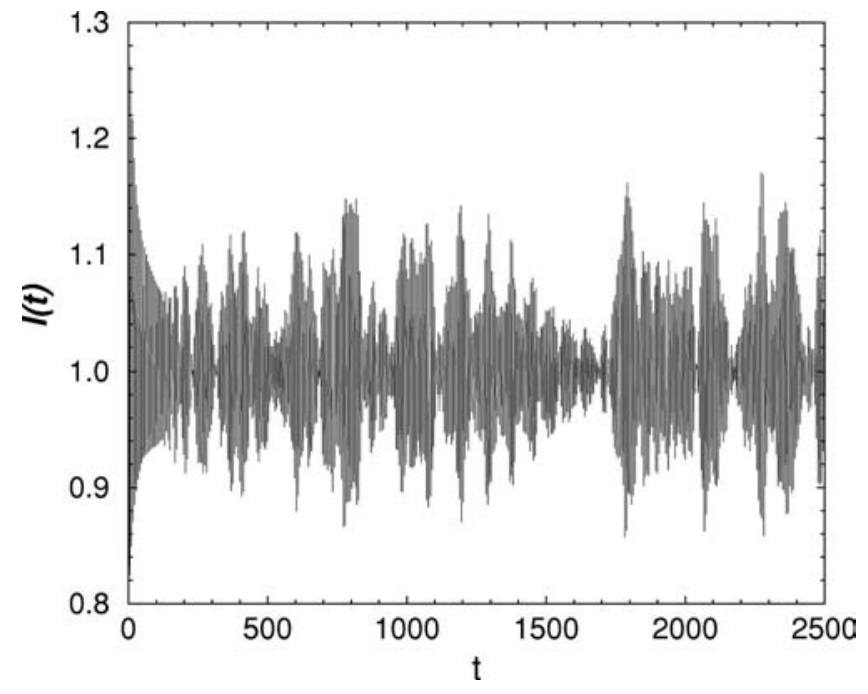

FIG. 2. Evolution of the kink width, in dimensionless units, obtained from numerical simulations starting from a free, deformed kink, with $u(0)=0, l(0)=1$ and $i(0)=0.3$. The parameters of the discretization are $L=100, \Delta x=0.1$, and $\Delta t=0.01$.

to the excitation of the internal mode, exhibiting oscillations in the kink width very close to those predicted by the $\mathrm{CC}$ approach. We then turned to the SG case, and we found that the oscillations are not correctly described by the $\mathrm{CC}$ equations. In Fig. 2 we can see the evolution of the kink width computed from numerical simulation of the SG system, with $l(0)=1, u(0)=0$, and $l(0)=0.3$. It is clear that $l(t)$ is not a simple oscillatory function as in the $\phi^{4}$ case, and Fourier analysis yields the following frequencies: $\omega_{d}=0.9983, \tilde{\omega}_{1}$ $=1.0034, \quad \tilde{\omega}_{2}=1.0083, \quad \tilde{\omega}_{3}=1.0184, \quad \tilde{\omega}_{4}=1.0335, \quad \tilde{\omega}_{5}$ $=1.0511, \tilde{\omega}_{6}=1.0712, \tilde{\omega}_{7}=1.0963$. As before, all these frequencies are very close to the discreteness induced internal mode and the first few radiational modes, with no evidence supporting the existence of the internal quasimode above the phonon band edge.

\section{CONCLUSIONS}

The first conclusion of this work is that the existence of the proposed internal quasimode of the SG kink above the phonon band edge [8] is very unlikely. We have found neither resonances nor long-lived kink width oscillations arising from such a mode when the kink is subject to ac driving or to initial deformations, respectively. We are confident that we have carefully explored all the range of relevant frequencies, as we have accurately detected all possible resonances within that range. In addition, the comparison with the phenomenology observed for the $\phi^{4}$ kink, which possesses a true internal mode, shows that if the SG kink would have such an internal mode, we would have detected its influence through numerical simulations as for the $\phi^{4}$ equation $[6,18]$. In fact, now that we have seen that radiation modes are parametrically excited by their corresponding half-frequency drivings, we believe we can suggest an explanation for the results of Boesch and Willis. Notice that they used Eq. (5) to calculate the lower phonon frequency, obtaining that $\omega_{1}$ $=\sqrt{1+(2 \pi / 1000)^{2}}=1.00002$, whereas the internal "quasimode" frequency that they found in the numerical simula- 
tions was $\omega_{s}=1.004$. As those two values were largely different, they concluded that the quasimode was well above the lowest phonon frequency and different from it. However, as we have discussed in the preceding sections, they used initial conditions in which the initial kink velocity was not zero; in this case, it can be easily shown that the corresponding frequencies of the phonons are given by

$$
\bar{\omega}_{k}=\frac{\omega_{k}-k u(0)}{\sqrt{1-u^{2}(0)}}, \quad \omega_{k}=\sqrt{1+k^{2}} .
$$

If we now insert the parameters used in [8] to perform a numerical experiment similar to the one discussed here, we find $\omega_{1}=1.004$, i.e., the resonance observed by Boesch and Willis took place in fact with the lowest frequency phonon in the presence of a moving kink and not with any internal quasimode.

The second conclusion of the present Rapid Communication relates to the recently proposed internal modes induced by perturbations, in our case by discreteness [12-14]. By the two different types of numerical simulations used in our research, we have detected the internal mode bifurcating from the edge of the phonon band (of the continuum problem). The frequency of that mode agrees qualitatively very well with the one predicted in [14]. Therefore, our research fully confirms the results of those papers and, in addition, shows that these perturbation induced internal modes behave very much like intrinsic ones. Our result, along with the finding of similar internal modes in other nonintegrable SG systems [19], strongly supports the generality of the phenomenon predicted in [13]. Finally, the fact that we are able to detect this mode reinforces our previous conclusion of the nonexistence of the internal quasimode, as indeed the SG kink responds to the ac driving or to deformations as it should if it had an internal mode, but for the discreteness induced one only. We thus believe that the present work definitely rules out the possibility of internal modes or "quasimodes" of SG kinks close to and above the lower phonon band edge.

\section{ACKNOWLEDGMENTS}

We thank Yuri Kivshar and Yuri Gaididei for discussions. Work at GISC (Leganés) has been supported by DGESIC (Spain) Grant No. PB96-0119. Travel between Bayreuth and Madrid has been supported by "Acciones Integradas Hispano-Alemanas,' a joint program of DAAD (Az. 314AI) and DGESIC.
[1] A. C. Scott, Nonlinear Science (Oxford University, Oxford, 1999).

[2] Yu. S. Kivshar and B. A. Malomed, Rev. Mod. Phys. 61, 763 (1989).

[3] A. Sánchez and A. R. Bishop, SIAM Rev. 40, 579 (1998).

[4] M. Peyrard and D. K. Campbell, Physica D 9, 33 (1983); D. K. Campbell, J. F. Schonfeld, and C. A. Wingate, ibid. 9, 1 (1983); D. K. Campbell, M. Peyrard, and P. Sodano, ibid. 19, 165 (1986).

[5] Yu. S. Kivshar, F. Zhang, and L. Vázquez, Phys. Rev. Lett. 67, 1177 (1991); Phys. Rev. A 46, 5214 (1992).

[6] N. R. Quintero, A. Sánchez, and F. G. Mertens, Phys. Rev. Lett. 84, 871 (2000).

[7] S. Habib, G. Lythe, and F. G. Mertens (unpublished).

[8] R. Boesch and C. R. Willis, Phys. Rev. B 42, 2290 (1990).

[9] M. J. Rice, Phys. Rev. B 28, 3587 (1983).

[10] As a few examples, see P. Tchofo Dinda, R. Boesch, E. Coquet, and C. R. Willis, Phys. Rev. B 46, 3311 (1992); Zhang Fei, Vladimir V. Konotop, Michel Peyrard, and Luis Vázquez, Phys. Rev. E 48, 548 (1993); Fei Zhang, Phys. Rev. E 54,
4325 (1996); Physica D 110, 51 (1997).

[11] E. Majerníková, Yu. B. Gaididei, and O. M. Braun, Phys. Rev. E 52, 1241 (1995).

[12] O. M. Braun, Yu. S. Kivshar, and M. Peyrard, Phys. Rev. E 56, 6050 (1997).

[13] Yu. S. Kivshar, D. E. Pelinovsky, T. Cretegny, and M. Peyrard, Phys. Rev. Lett. 80, 5032 (1998).

[14] P. G. Kevrekidis and C. K. R. T. Jones, Phys. Rev. E 61, 3114 (2000).

[15] F. G. Mertens, H. J. Schnitzer, and A. R. Bishop, Phys. Rev. B 56, 2510 (1997).

[16] N. R. Quintero and A. Sánchez, Eur. Phys. J. B 6, 133 (1998).

[17] W. A. Strauss and L. Vázquez, J. Comput. Phys. 28, 271 (1978).

[18] N. R. Quintero, A. Sánchez, and F. G. Mertens (unpublished). We note that in [11] only solutions for a special, restricted set of initial conditions were found.

[19] S. F. Mingaleev, Yu. B. Gaididei, E. Majerníková, and S. Shpyrko, Phys. Rev. E 61, 4454 (2000). 